Article

\title{
Chaos Synchronization of Nonlinear Fractional Discrete Dynamical Systems via Linear Control
}

\author{
Baogui Xin *, ${ }^{+}, \mathrm{Li} \mathrm{Liu}^{\dagger}$, Guisheng $\mathrm{Hou}^{\dagger}$ and Yuan $\mathrm{Ma}^{\dagger}$ \\ Nonlinear Science Center, School of Economics and Management, Shandong University of Science and \\ Technology, Qingdao 266590, China; sunshineli1993@163.com (L.L.); ieeehou@163.com (G.H.); \\ dodomy@163.com (Y.M.) \\ * Correspondence: xin@tju.edu.cn; Tel.: +86-532-8605-7025 \\ + These authors contributed equally to this work.
}

Received: 21 May 2017; Accepted: 7 July 2017; Published: 11 July 2017

\begin{abstract}
By using a linear feedback control technique, we propose a chaos synchronization scheme for nonlinear fractional discrete dynamical systems. Then, we construct a novel 1-D fractional discrete income change system and a kind of novel 3-D fractional discrete system. By means of the stability principles of Caputo-like fractional discrete systems, we lastly design a controller to achieve chaos synchronization, and present some numerical simulations to illustrate and validate the synchronization scheme.
\end{abstract}

Keywords: fractional discrete systems; linear feedback control; chaos synchronization; fractional discrete income change systems

\section{Introduction}

Over the last decades, increasing interest has been shown in fractional differential calculus, which has been successfully applied to various fields, such as biology [1], fluid mechanics [2], materials science [3], physics [4], and economics [5]. Recently, fractional discrete calculus has gained more and more attention and a lot of interesting results have emerged in mathematics [6-13], medical science [14], physics [15,16], and so on.

Chaos synchronization has been a hot topic [17-19]. There are many synchronization schemes for fractional differential systems, such as synchronization via the linear control technique [20], synchronization via the adaptive sliding mode [21], projective synchronization via single sinusoidal coupling [22], hybrid chaos synchronization with a robust method [23], synchronization with activation feedback control [24], synchronization via a scalar transmitted signal [25], adaptive synchronization via a single driving variables [26], synchronization via novel active pinning controls [27]. In fact, most mentioned synchronization schemes of fractional differential systems can be used in the synchronization of fractional discrete dynamical systems.

To date, only four synchronization schemes have been proposed for fractional discrete dynamical systems, as follows: chaos synchronization based on the stability condition [8], chaos synchronization with a nonlinear coupling method [28], and chaos synchronization with linear coupling strength [29]; exact synchronization is established by designing a step-by-step delayed observer [30]. Just as the linear feedback control technique can be employed to achieve the synchronization of fractional differential systems, one may spontaneously want to know whether or not it can be used to obtain the synchronization of fractional discrete dynamical systems.

Linear feedback control is an economic, robust and easily implemented control technique available for chaos synchronization [31]. Odibat et al. [20] designed a kind of synchronization scheme for three-dimensional chaotic fractional-order systems. Xin et al. employed the linear feedback control technique to design projective synchronization schemes for chaotic discrete dynamical systems [32] and 
fractional differential dynamical systems [33-35], respectively. In this paper, the linear feedback control technique will be applied to achieve the synchronization of fractional discrete dynamical systems. Comparing the aforementioned four synchronization schemes for fractional discrete dynamical systems [8,28-30], the linear feedback control technique is not only easier to design and implement but is also more intuitive for the simplest linear stability theory of fractional discrete dynamical systems.

The remainder of this paper is organized as follows: in Section 2, preliminaries are presented. In Section 3, a synchronization scheme for n-dimensional nonlinear fractional discrete dynamical systems is proposed. The proposed synchronization scheme is applied to novel 1-D income change systems in Section 4, and novel 3-D fractional discrete dynamical systems in Section 5. Finally, the paper is concluded in Section 6.

\section{Preliminaries}

Some definitions of fractional discrete time calculus are introduced as follows.

Definition 1. (Atici and Eloe [6]). Let $x: \mathbb{N}_{a} \rightarrow \mathbb{R}$ and $0<v$ be given. Then the fractional sum of $v$ order is defined by

$$
\Delta_{a}^{-v} x(t):=\frac{1}{\Gamma(v)} \sum_{s=a}^{t-v}(t-\sigma(s))^{(v-1)} x(s), \quad t \in \mathbb{N}_{a+v}
$$

where $a$ is the start point, $\mathbb{N}_{a}=\{a, a+1, a+2, \ldots\}$ denotes the isolated time scale, $\sigma(s)=s+1$, and $t^{(v)}$ is the falling function defined as

$$
t^{(v)}=\frac{\Gamma(t+1)}{\Gamma(t+1-v)}
$$

Definition 2. (Abdeljawad [36]). For $0<v, v \notin \mathbb{N}$ and $x(t)$ defined on $\mathbb{N}_{a}$, the Caputo-like delta difference is defined by

$$
{ }^{C} \Delta_{a}^{v} x(t):=\Delta_{a}^{-(m-v)} \Delta^{m} x(t)=\frac{1}{\Gamma(m-v)} \sum_{s=a}^{t-(m-v)}(t-\sigma(s))^{(m-v-1)} \Delta_{s}^{m} x(s),
$$

where $t \in \mathbb{N}_{a+m-v}$ and $m=[v]+1$.

Theorem 1. (Chen et al. [37]). For the delta fractional difference equation

$$
\left\{\begin{array}{c}
{ }^{C} \Delta_{a}^{v} x(t)=f(t+v-1, x(t+v-1)), \\
\Delta^{k} x(a)=x_{k}, \quad k=0, \ldots, m-1,
\end{array}\right.
$$

the equivalent discrete integral equation can be obtained as

$$
x(t)=x_{0}(t)+\frac{1}{\Gamma(v)} \sum_{s=a+m-v}^{t-v}(t-\sigma(s))^{(v-1)} f(s+v-1, x(s+v-1)), \quad t \in \mathbb{N}_{a+m}
$$

where the initial iteration $x_{0}(t)$ reads

$$
x_{0}(t)=\sum_{k=0}^{m-1} \frac{(t-a)^{(k)}}{\Gamma(k+1)} \Delta^{k} x(a)
$$

Remark 1. If $0<v<1$ and $a=0$, then Equation (5) can be rewritten as

$$
x(t)=x(0)+\frac{1}{\Gamma(v)} \sum_{s=1-v}^{t-v}(t-\sigma(s))^{(v-1)} f(s+v-1, x(s+v-1)), \quad t \in \mathbb{N}_{1}
$$


Theorem 2. (Baleanu et al. [38]). Equation (4) is asymptotically stable if $x=0$ is an equilibrium point of Equation (4) and $x(t+v-1) f(t+v-1, x(t+v-1))<0$ holds for all $t \in \mathbb{N}_{a+1-v}$.

\section{A Synchronization Scheme of Nonlinear Fractional Discrete Dynamical Systems}

Consider the following nonlinear fractional discrete dynamical system:

$$
{ }^{C} \Delta_{a}^{v} x(t)=L(x(t+v-1))+N(x(t+v-1)), \quad 0<v \leq 1, \quad x(a)=c, \quad t \in(\mathbb{N})_{a+1-v}
$$

where ${ }^{C} \Delta_{a}^{v}$ is the left Caputo-like delta difference, $x=\left(x_{1}, x_{2}, \cdots, x_{n}\right)^{T} \in \mathbb{R}^{n}$ is an n-dimensional state vector of system (8), and $L, N: \mathbb{R}^{n} \rightarrow \mathbb{R}^{n}$ are linear and nonlinear functions of states, respectively.

Correspondingly, one may construct the following nonlinear fractional discrete dynamical system:

$$
{ }^{C} \Delta_{a}^{v} y(t)=L(y(t+v-1))+N(x(t+v-1))+u(t+v-1), \quad 0<v \leq 1, \quad t \in(\mathbb{N})_{a+(1-v)},
$$

where $y=\left(y_{1}, y_{2}, \cdots, y_{n}\right)^{T} \in \mathbb{R}^{n}$ is an $\mathrm{n}$-dimensional state vector of system (9), and $u=\left(u_{1}, u_{2}, \cdots, u_{n}\right)^{T} \in \mathbb{R}^{n}$ is an n-dimensional linear state error feedback controller.

Defining the following synchronization error between the master system (8) and the slave system (9):

$$
e(t)=y(t)-x(t)
$$

The linear state error feedback controller $u(t)$ can be defined as follows:

$$
u(t)=\hat{A} e(t)
$$

where $\hat{A}$ is an $n \times n$ linear constant matrix. Subtracting (8) from (9), the following error system can be obtained:

$$
{ }^{C} \Delta_{a}^{v} e(t)={ }^{C} \Delta_{a}^{v} y(t)-{ }^{C} \Delta_{a}^{v} x(t)=A e(t+v-1), \quad 0<v \leq 1, \quad t \in(h \mathbb{N})_{a+(1-v-1)}
$$

where $A=L+\hat{A}$ is an $n \times n$ linear constant matrix. Obviously, the original point is the fixed point of system (12), so one can directly obtain the following theorem by means of Theorem 2.

Theorem 3. If $e^{T}(t+v-1) A e(t+v-1)<0$ holds for all $t \in \mathbb{N}_{a+1-v}$, then the synchronization error $e(t)$ is asymptotically stable and $\lim _{t \rightarrow \infty} e(t)=0$, i.e., systems (8) and (9) achieve projective synchronization.

\section{Application to the Fractional Discrete Dynamical Income Change System}

\subsection{A Fractional Discrete Dynamical Income Change System}

Puu and Sushko [39] presented the following chaotic discrete dynamical income system with cubic nonlinearity:

$$
x(t+1)=\varphi x(t)-(\varphi+1)(x(t))^{3}
$$

with $x$ denoting the change of income between the two previous periods, and $\varphi \geq 0$.

One can introduce the discrete fractional calculus into system (13) as follows:

$$
{ }^{\mathrm{C}} \Delta_{a}^{v} x(t)=\varphi x(t+v-1)-(\varphi+1)(x(t+v-1))^{3} .
$$

Figure 1a shows solutions of system (14), which are chaotic. Figure $1 \mathrm{~b}$ is a superposed diagram of bifurcation and the largest Lyapunov exponents with $\varphi \in[0.6,1.4]$ and $v=0.7$. When $\varphi=1.4$, the largest Lyapunov exponent $\lambda=0.386$ which means that chaos occurs in system (14). 


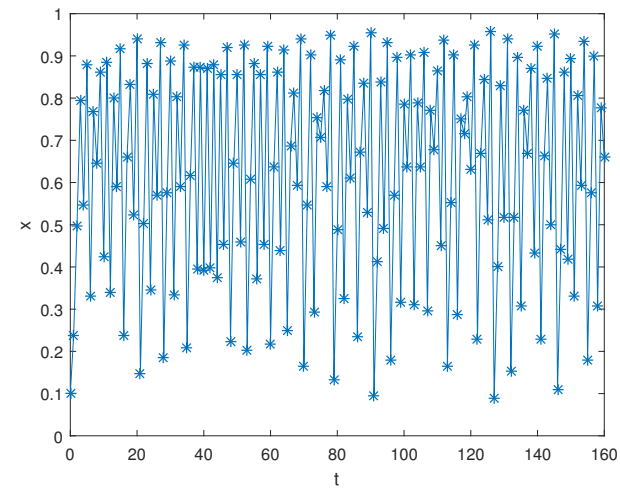

(a)

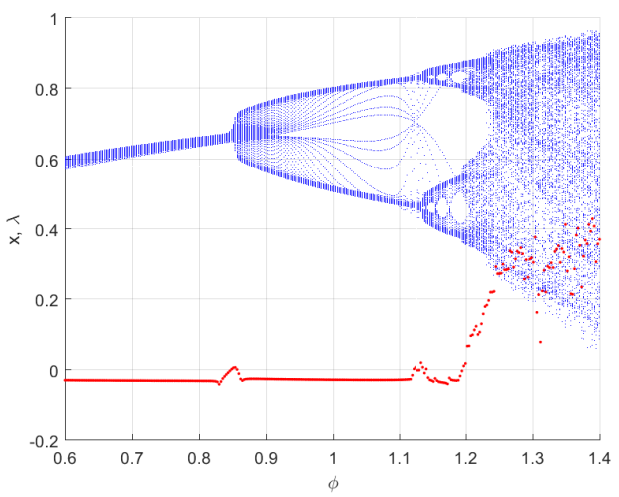

(b)

Figure 1. The chaos in system (14) with $v=0.7$ and $x(0)=0.1$. (a) The chaotic solutions with $\varphi=1.4$; (b) Bifurcation (blue) and the largest Lyapunov exponents (red) with $\varphi$ varying from 0.6 to 1.4 .

\subsection{A Synchronization Scheme of Fractional Discrete Dynamical Income Change Systems}

One can regard system (14) as the master system and construct the following slave system (denoted by the subscript $s$ ):

$$
{ }^{\mathrm{C}} \Delta_{a}^{v} x_{S}(t)=\varphi x_{s}(t+v-1)-(\varphi+1)(x(t+v-1))^{3}+u(t+v-1) .
$$

where $u(t+v-1)$ is the linear state error feedback controller.

Proposition 1. If the following control laws hold, the master-slave systems (14) and (15) will finally achieve global projective synchronization for any initial condition:

$$
u(t)=\kappa\left(x_{s}(t+v-1)-x(t+v-1)\right), \quad \kappa+\varphi<0 .
$$

Proof. One can define the synchronization errors between the master-slave systems (14) and (15) as follows:

$$
e(t)=x_{s}(t)-x(t) .
$$

Subtracting system (14) from (15), one may get the following error system:

$$
{ }^{\mathrm{C}} \Delta_{a}^{v} e(t)=\varphi e(t+v-1)+u(t+v-1) .
$$

Substituting Equation (16) into the error system (18), the following error system can be obtained:

$$
{ }^{\mathrm{C}} \Delta_{a}^{v} e(t)=(\kappa+\varphi) e(t+v-1),
$$

which has a trivial solution $e(t)=0$, and the following equation holds:

$$
e^{T}(t+v-1) A e(t+v-1)=(\kappa+\varphi)(e(t+v-1))^{2}<0 .
$$

With Theorem 2, one can find that system (19) is asymptotically stable, i.e., the master system (14) and the slave system (15) finally achieve synchronization, as shown in Figure 2.

The Proposition 1 is thus proved. 


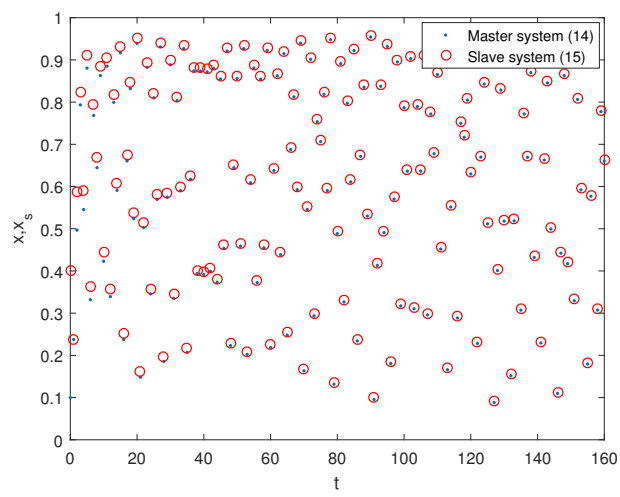

(a)

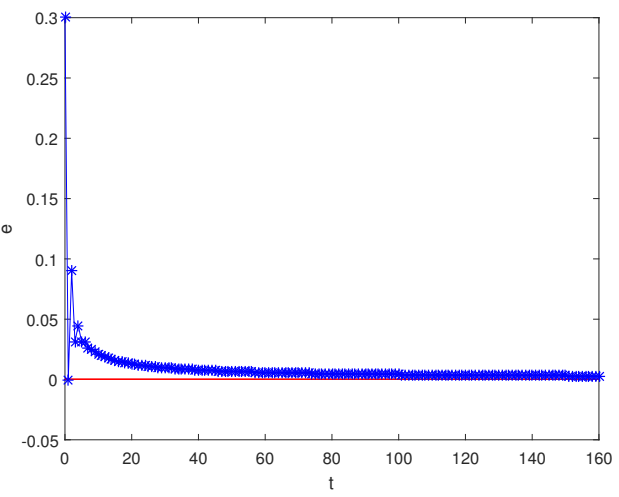

(b)

Figure 2. Chaos synchronization of systems (14) and (15) with $v=0.7, \varphi=1.4, \kappa=-2.4, x(0)=0.1$ and $y(0)=0.4$. (a) Chaos synchronization of the master system (14) and the slave system (15); (b) Error evolution.

\section{Application to the Novel Three-Dimensional Fractional Discrete Dynamical System}

\subsection{A Novel Three-Dimensional Fractional Discrete Dynamical System}

In this section, we present a novel three-dimensional fractional chaotic discrete dynamical system as follows:

$$
\left\{\begin{array}{l}
x_{n+1}=-x_{n}+\theta \tan \left(y_{n}\right), \\
y_{n+1}=x_{n}-\theta z_{n} \\
z_{n+1}=-\theta x_{n}+y_{n},
\end{array}\right.
$$

where $\theta \geq 0$. By introducing the discrete fractional calculus into system (21), one may get:

$$
\left\{\begin{array}{l}
{ }^{C} \Delta_{a}^{v} x(t)=-x(t+v-1)+\theta \tan (y(t+v-1)), \\
{ }^{C} \Delta_{a}^{v} y(t)=x(t+v-1)-\theta z(t+v-1), \\
{ }^{C} \Delta_{a}^{v} z(t)=-\theta x(t+v-1)+y(t+v-1),
\end{array}\right.
$$

Figure 3 a shows a chaotic attractor of system (22), and Figure $3 b$ shows a superposed diagram of bifurcation and the largest Lyapunov exponents with $\theta \in[0,0.1]$ and $v=0.9$. When $\theta=0.1$, the largest Lyapunov exponent $\lambda=0.066$ which also implies that chaos exists in system (22).

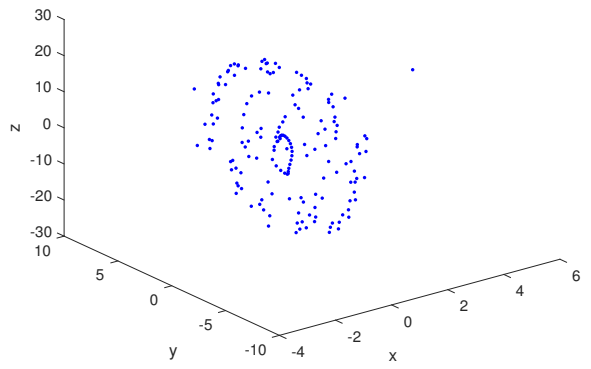

(a)

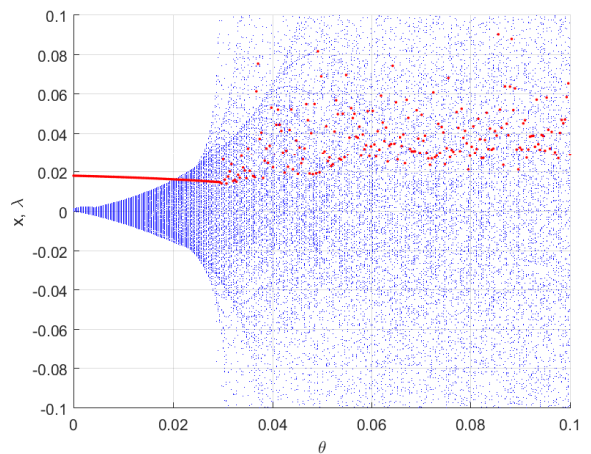

(b)

Figure 3. The chaos in system (14) with $v=0.9, x(0)=0.3, y(0)=0.4$ and $z(0)=0.2$. (a) The chaotic solutions with $\theta=0.1$; (b) Bifurcation (blue) and the largest Lyapunov exponents (red) with $\theta$ varying from 0 to 0.1 . 


\subsection{A Synchronization Scheme of the Novel Three-Dimensional Fractional Discrete Dynamical Systems}

System (14) can be regarded as the master system, and the slave system (denoted by the subscript $s$ ) can be constructed as follows:

$$
\left\{\begin{array}{l}
{ }^{C} \Delta_{a}^{v} x_{s}(t)=-x_{s}(t+v-1)+\theta \tan (y(t+v-1))+u_{x}(t+v-1), \\
{ }^{C} \Delta_{a}^{v} y_{s}(t)=x_{s}(t+v-1)-\theta z_{s}(t+v-1)+u_{y}(t+v-1), \\
{ }^{C} \Delta_{a}^{v} z_{s}(t)=-\theta x_{s}(t+v-1)+y_{s}(t+v-1)+u_{z}(t+v-1),
\end{array}\right.
$$

where $u_{x}(t+v-1), u_{y}(t+v-1)$ and $u_{z}(t+v-1)$ are linear state error feedback controllers.

Proposition 2. If the following control laws hold, the master-slave systems (22) and (23) will finally achieve global projective synchronization for any initial condition:

$$
\left\{\begin{array}{l}
u_{x}(t+v-1)=z_{s}(t+v-1)-z(t+v-1) \\
u_{y}(t+v-1)=x(t+v-1)-x_{s}(t+v-1) \\
u_{z}(t+v-1)=(\theta-1)\left(y_{s}(t+v-1)-y(t+v-1)\right) .
\end{array}\right.
$$

Proof. One can define the following synchronization errors between the master-slave systems (22) and (23):

$$
\left\{\begin{array}{l}
e_{x}(t)=x_{s}(t)-x(t) \\
e_{y}(t)=y_{s}(t)-y(t) \\
e_{z}(t)=z_{s}(t)-z(t)
\end{array}\right.
$$

Subtracting system (22) from (23), one may get the following error system:

$$
\left\{\begin{array}{l}
{ }^{C} \Delta_{a}^{v} e_{x}(t)=-e_{x}(t+v-1)+u_{x}(t+v-1), \\
{ }^{C} \Delta_{a}^{v} e_{y}(t)=e_{x}(t+v-1)-\theta e_{z}(t+v-1)+u_{y}(t+v-1), \\
{ }^{C} \Delta_{a}^{v} e_{z}(t)=-\theta e_{x}(t+v-1)+e_{y}(t+v-1)+u_{z}(t+v-1) .
\end{array}\right.
$$

Substituting Equation (24) into the error system (26), the following error system can be obtained:

$$
\left\{\begin{array}{l}
{ }^{C} \Delta_{a}^{v} e_{x}(t)=-e_{x}(t+v-1)+e_{z}(t+v-1), \\
{ }^{C} \Delta_{a}^{v} e_{y}(t)=-\theta e_{z}(t+v-1), \\
{ }^{C} \Delta_{a}^{v} e_{z}(t)=-\theta\left(e_{x}(t+v-1)-e_{y}(t+v-1)\right),
\end{array}\right.
$$

which has a trivial solution $e(t)=0$, and the following equation holds.

$$
\begin{aligned}
e^{T}(t+v-1) A e(t+v-1) & =\left(\begin{array}{c}
e_{x}(t+v-1) \\
e_{y}(t+v-1) \\
e_{z}(t+v-1)
\end{array}\right)^{T}\left(\begin{array}{c}
-e_{x}(t+v-1)+e_{z}(t+v-1) \\
-\theta e_{z}(t+v-1) \\
-\theta\left(e_{x}(t+v-1)-e_{y}(t+v-1)\right)
\end{array}\right) \\
& =-\left(e_{x}(t+v-1)\right)^{2}<0 .
\end{aligned}
$$

With Theorem 2, one can find that the system (27) is asymptotically stable, i.e., the master system (22) and the slave system (23) finally achieve synchronization, as shown in Figure 4.

This concludes the proof. 


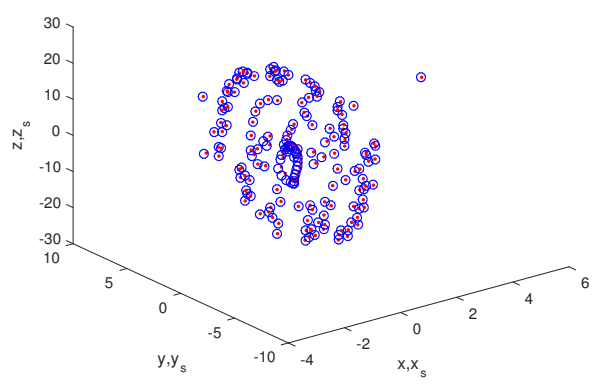

(a)

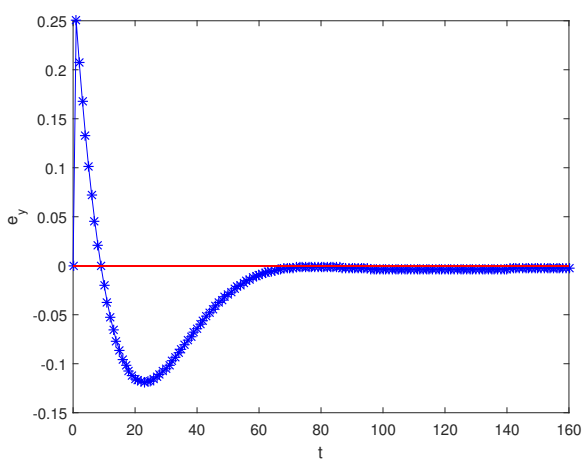

(c)

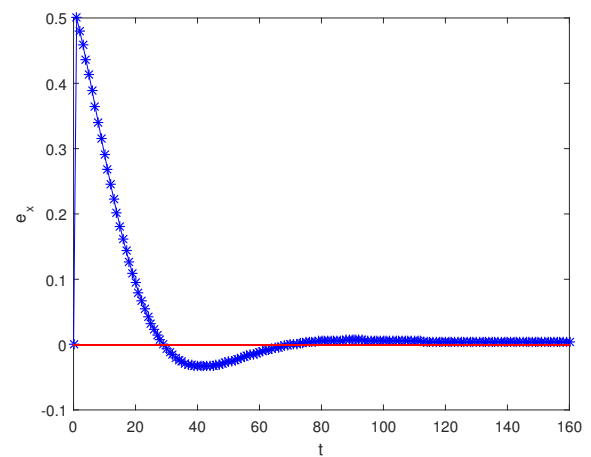

(b)

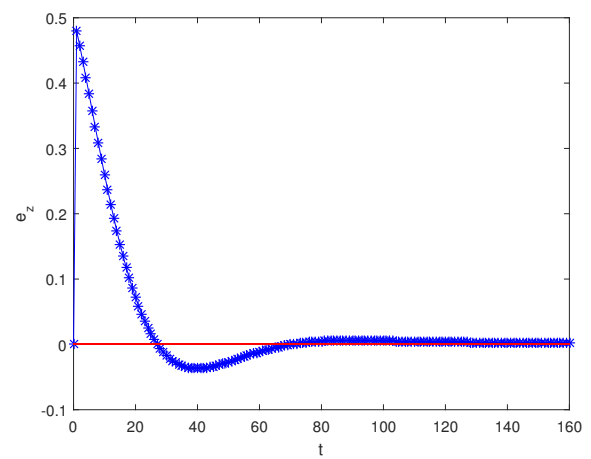

(d)

Figure 4. Chaos synchronization of systems (22) and (23) with $v=0.9, \theta=0.1, x(0)=0.3, y(0)=0.4$, $z(0)=0.2$ and $x_{s}(0)=y_{s}(0)=z_{s}(0)=0.1$. (a) Chaos synchronization of the master system (22) (blue circles) and the slave system (23) (red dots); (b) Evolution of error $e_{x}$; (c) Evolution of error $e_{y}$; (d) Evolution of error $e_{z}$.

\section{Conclusions}

- $\quad$ The proposed 1-D fractional discrete income change system and a kind of novel 3-D fractional chaotic discrete system are employed to implement some interesting numerical simulations, which coincide well with the mentioned results.

- The proposed chaos synchronization scheme via the linear feedback control technique is very robust and easy to implement efficiently, and should have additional interesting applications in the future, such as secure communications, information storage, message identification, encryption and decryption.

- It is necessary for us to employ other control techniques, such as active control, sliding mode control, and pinning control, to achieve chaos synchronization of nonlinear fractional discrete dynamical systems.

- It will be challenging and interesting to expand the mentioned results to study the anti-synchronization or finite-time synchronization of the fractional discrete dynamic systems via the linear feedback control technique or others.

Acknowledgments: The authors would like to express sincere gratitude to the referees for their valuable suggestions and comments. The work is supported by the National Social Science Foundation of China (No. 16FJY008).

Author Contributions: All authors jointly worked on deriving the results and wrote the paper. All authors have read and approved the final manuscript.

Conflicts of Interest: The authors declare no conflict of interest. 


\section{References}

1. Craiem, D.; Magin, R. Fractional order models of viscoelasticity as an alternative in the analysis of red blood cell (RBC) membrane mechanics. Phys. Biol. 2010, 7, 013001.

2. Jiang, Y.; Qi, H.; Xu, H.; Jiang, X. Transient electroosmotic slip flow of fractional Oldroyd-B fluids. Microfluid. Nanofluid. 2017, 21, 7, doi:10.1007/s10404-016-1843-x.

3. Xu, H.; Jiang, X. Creep constitutive models for viscoelastic materials based on fractional derivatives. Comput. Math. Appl. 2017, 73, 1377-1384.

4. Herrmann, R. Fractional Calculus: An Introduction for Physicists; World Scientific: Singapore, 2014.

5. Xin, B.; Zhang, J. Finite-time stabilizing a fractional-order chaotic financial system with market confidence. Nonlinear Dyn. 2015, 79, 1399-1409.

6. Atici, F.; Eloe, P. Initial value problems in discrete fractional calculus. Proc. Am. Math. Soc. 2009, 137, 981-989.

7. Abu-Saris, R.; Al-Mdallal, Q. On the asymptotic stability of linear system of fractional-order difference equations. Fract. Calc. Appl. Anal. 2013, 16, 613-629.

8. Wu, G.; Baleanu, D.; Xie, H.; Chen, F. Chaos synchronization of fractional chaotic maps based on the stability condition. Physica A 2016, 460, 374-383.

9. Wyrwas, M.; Mozyrska, D.; Girejko, E. Stability of discrete fractional-order nonlinear systems with the nabla Caputo difference. IFAC Proc. Vol. 2013, 46, 167-171.

10. Mozyrska, D.; Wyrwas, M. Explicit criteria for stability of fractional h-difference two-dimensional systems. Int. J. Dyn. Control 2016, 2016, 1-6.

11. Mohan, J.; Deekshitulu, G. Fractional order difference equations. Int. J. Differ. Equ. 2012, 2012, 1-11, doi:10.1155/2012/780619.

12. Dassios, I. Stability and robustness of singular systems of fractional nabla difference equations. Circuits Syst. Signal Process. 2017, 36, 49-64.

13. Diblík, J.; Fečkan, M.; Pospíšil, M. Nonexistence of periodic solutions and S-asymptotically periodic solutions in fractional difference equations. Appl. Math. Comput. 2015, 257, 230-240.

14. Atıc1, F.M.; Şengl, S. Modeling with fractional difference equations. J. Math. Anal. Appl. 2010, 369, 1-9.

15. Hu, T. Discrete Chaos in Fractional Henon Map. Appl. Math. 2014, 5, 2243-2248.

16. Wu, G.; Baleanu, D. Discrete fractional logistic map and its chaos. Nonlinear Dyn. 2014, 75, $283-287$.

17. Chen, D.; Zhao, W.; Sprott, J.; Ma, X. Application of Takagi-Sugeno fuzzy model to a class of chaotic synchronization and anti-synchronization. Nonlinear Dyn. 2013, 73, 1495-1505.

18. Chen, D.; Shi, L.; Chen, H.; Ma, X. Analysis and control of a hyperchaotic system with only one nonlinear term. Nonlinear Dyn. 2012, 67, 1745-1752.

19. Chen, D.; Zhang, R.; Sprott, J.; Ma, X. Synchronization between integer-order chaotic systems and a class of fractional-order chaotic system based on fuzzy sliding mode control. Nonlinear Dyn. 2012, 70, 1549-1561.

20. Odibat, Z.; Corson, N.; Aziz-Alaoui, M.; Bertelle, C. Synchronization of chaotic fractional-order systems via linear control. Int. J. Bifurc. Chaos 2010, 20, 81-97.

21. Shao, S.; Chen, M.; Yan, X. Adaptive sliding mode synchronization for a class of fractional-order chaotic systems with disturbance. Nonlinear Dyn. 2016, 83, 1855-1866.

22. Li, C.; Zhang, M.; Zhou, F.; Yang, X. Projective synchronization for a fractional-order chaotic system via single sinusoidal coupling. Opt. Int. J. Light Electron Opt. 2016, 127, 2830-2836.

23. Ouannas, A.; Azar, A.; Vaidyanathan, S. A robust method for new fractional hybrid chaos synchronization. Math. Methods Appl. Sci. 2017, 40, 1804-1812.

24. Wang, X.; Song, J. Synchronization of the fractional order hyperchaos Lorenz systems with activation feedback control. Commun. Nonlinear Sci. Numer. Simul. 2009, 14, 3351-3357.

25. Lu, J. Synchronization of a class of fractional-order chaotic systems via a scalar transmitted signal. Chaos Solitons Fract. 2006, 27, 519-525.

26. Zhang, R.; Yang, S. Adaptive synchronization of fractional-order chaotic systems via a single driving variable. Nonlinear Dyn. 2011, 66, 831-837.

27. Pan, L.; Zhou, W.; Fang, J.; Li, D. Synchronization and anti-synchronization of new uncertain fractional-order modified unified chaotic systems via novel active pinning control. Commun. Nonlinear Sci. Numer. Simul. 2010, 15, 3754-3762. 
28. Wu, G.; Baleanu, D. Chaos synchronization of the discrete fractional logistic map. Signal Process. 2014, 102, 96-99.

29. Liu, Y. Chaotic synchronization between linearly coupled discrete fractional Hénon maps. Indian J. Phys. 2016, 90, 313-317.

30. Kassim, S.; Hamiche, H.; Djennoune, S.; Bettayeb, M. A novel secure image transmission scheme based on synchronization of fractional-order discrete-time hyperchaotic systems. Nonlinear Dyn. 2017, 88, 2473.

31. Wu, X.; Chen, G.; Cai, J. Chaos synchronization of the master-slave generalized Lorenz systems via linear state error feedback control. Physica D 2007, 229, 52-80.

32. Xin, B.; $\mathrm{Wu}, \mathrm{Z}$. Projective synchronization of chaotic discrete dynamical systems via linear state error feedback control. Entropy 2015, 17, 2677-2687.

33. Xin, B.; Chen, T.; Liu, Y. Projective synchronization of chaotic fractional-order energy resources demand-supply systems via linear control. Commun. Nonlinear Sci. Numer. Simul. 2011, 16, 4479-4486.

34. Xin, B.; Chen, T.; Liu, Y. Synchronization of chaotic fractional-order WINDMI systems via linear state error feedback control. Math. Probl. Eng. 2010, 2010, 1-10, doi:10.1155/2010/859685.

35. Xin, B.; Chen, T. Projective synchronization of $N$-dimensional chaotic fractional-order systems via linear state error feedback control. Discret. Dyn. Nat. Soc. 2012, 2012, 1-10, doi:10.1155/2012/191063.

36. Abdeljawad, T. On Riemann and Caputo fractional differences. Comput. Math. Appl. 2011, 62, 1602-1611.

37. Chen, F.; Luo, X.; Zhou, Y. Existence Results for Nonlinear Fractional Difference Equation. Adv. Differ. Equ. 2011, 2011, 713201, doi:10.1155/2011/713201.

38. Baleanu, D.; Wu, G.; Bai, Y.; Chen, F. Stability analysis of Caputo-Like discrete fractional systems. Commun. Nonlinear Sci. Numer. Simul. 2017, 48, 520-530.

39. Puu, T.; Sushko, I. A business cycle model with cubic nonlinearity. Chaos Solitons Fract. 2004, 19, 597-612.

(C) 2017 by the authors. Licensee MDPI, Basel, Switzerland. This article is an open access article distributed under the terms and conditions of the Creative Commons Attribution (CC BY) license (http:/ / creativecommons.org/licenses/by/4.0/). 